\title{
Evolución de la pobreza energéti- ca en Argentina durante el período 2002 - 2018. Oportunidades para las Energías Renovables
}

Rodrigo Durán ${ }^{1}$, Miguel Condorí2 2

Resumen:

En la presente comunicación se presenta la evaluación de un índice de pobreza energética para Argentina y sus aglomerados urbanos durante el periodo 2003 - 2018. El índice mantiene una definición empleada por la Unión Europea mediante la cual se compara el ingreso total familiar $y$ los gastos que realiza un hogar para acceder a las fuentes residenciales de energía secundaria. Para evaluar el indicador se ha construido una base de datos con información, poblacional, de consumo energético residencial y tarifaria provenientes de, ENARGAS, Ministerio de Energía de la Nación, CAMMESA y distribuidoras de energía eléctrica. Los resultados indican un amplio aumento de la pobreza energética durante el periodo 2015 - 2016 a nivel nacional, siendo de un $0,8 \%$ a un $15,1 \%$ y una baja cobertura de la Tarifa Social sobre los hogares en esta situación, (entre el $42,2 \%$ al $66,3 \%$, dependiendo del cumplimiento del requerimiento de titularidad del servicio energético). En este contexto, marcado por el aumento tarifario, el desarrollo de la matriz energética de base renovable para el sector residencial se presenta como una alternativa válida para acompañar las políticas de inclusión social - energética.

Palabras clave: Pobreza energética, Políticas energéticas, Energía e inclusión social

\section{Abstract:}

This paper presents the evaluation of an energy poverty index for Argentina and its urban agglomerates during the period $2003-2018$. The index complies with a definition used by the European Union,

\footnotetext{
${ }^{1}$ Instituto de Investigaciones en Energía no Convencional - Universidad Nacional de Salta. duran.cayon@gmail.com, 2 Instituto de Investigaciones en Energía no Convencional - Universidad Nacional de Salta. miguel.angel.condori@ gmail.com
} 
which compares the total family income and the expenses that makes a home to access residential secondary energy sources.

To evaluate the indicator, a database with data supplied by ENARGAS, the Ministry of Energy of the Nation, CAMMESA and electricity distributors has been built. The results indicate a large increase in energy poverty for Argentina during the period 2015-2016, from $0.8 \%$ to $15.1 \%$ and low coverage of the Social Tariff on households in this situation (between $42.2 \%$ to $66.3 \%$, which depends on compliance with the ownership requirement of the energy service). In this context, marked by the tariff increase, the development of the renewable base energy matrix for the residential sector is presented as a valid alternative to accompany the social and energy inclusion policies.

Keywords: Energy poverty, Energy policies, Energy and social inclusion

\section{Introducción}

El concepto de pobreza energética -entendido como la imposibilidad de un hogar a hacer frente a los requerimientos energéticos básicos (González-Eguino, 2015) y su estudio en el contexto de Argentina, resulta útil para la definición de políticas públicas de desarrollo energético con un enfoque humano y adecuado a los hogares más vulnerables (Bazilian et al., 2014). Así, este trabajo tiene como objetivo la estimación de un índice de pobreza energética en los hogares urbanos de Argentina para el periodo 2003 - 2018, al mismo tiempo que busca caracterizar las problemáticas transversales a los hogares en situación de pobreza energética en términos de su formalidad laboral, composición familiar, nivel educativo, confort térmico de la vivienda que ocupan y género, entre otros. Asimismo, el periodo definido permite observar los cambios en la intensidad con que actúa la pobreza energética en términos de la problemáticas económicas y decisiones políticas implementadas por los gobiernos de turno.

De manera similar al concepto de pobreza, las definiciones de pobreza energética vienen siendo trabajadas desde distintas perspectivas, las cuales son implícitamente fundamentadas mediante el concepto de desarrollo (Bouzarovski et al., 2012). En términos generales, pobreza energética refiere a la imposibilidad que tiene un hogar de cubrir los requerimientos energéticos considerados como básicos para el desarrollo y el mantenimiento de la vida digna (Okushima, 2016). Las diferentes interpretaciones a la que da lugar esta idea general y su adecuación al contexto en el cual se estima, han llevado a variaciones en torno a la definición de pobreza energética. Algunos autores ponen énfasis en los aspectos relativos a la desigualdad en el acceso y disponibilidad de los recursos energéticos (Pachauri and Spreng, 2004), la asequibilidad del recurso, o la definición de un estándar energético mínimo que debe ser cubierto para vivir confortablemente (Nussbaumer et al., 2012). Un indicador de pobreza energética ampliamente utilizado (Nussbaumer et al., 2012; Pachauri y Spreng, 2011), define a un ho- 
gar como pobre energético como aquel en el que se destina más del 10\% del total de sus ingresos para acceder a las fuentes energéticas. Se ha escogido a partir de la consideración de las características de las fuentes de datos.

\section{Materiales y Métodos}

En este trabajo, y por las características de las fuentes de datos públicos en Argentina, es posible la estimación de índice de pobreza energética basado en la definición de línea de pobreza energética. Así, un hogar será pobre energético cuando los gastos totales que realiza para poder acceder a las fuentes de energía de uso residencial son mayores al 10\% de sus ingresos totales.

Se emplean datos pertenecientes a la Encuesta Permanente de Hogares (EPH), relevada trimestralmente por el Instituto de Censos y Estadísticas de la Nación (INDEC), esta encuesta revisa aspectos económicos, laborales, de vivienda, acceso a servicios y relativos a la población en general (como los son las trayectorias educativas, composición familiar, acceso al sistema de salud entro otros). Si bien, al tratarse de una encuesta, los resultados de la EPH son afectados por un error estadístico, las descripciones, análisis y conclusiones a las que se arriba a partir de la EPH tienen un alto alcance y potencial explicativo con respecto a la población urbana del país y sus regiones.

Los aspectos relativos a la demanda energética y su costo para el sector residencial fueron estimados según fuentes oficiales y de acuerdo a la fuente de ener- gía. Así, para estimar el costo del acceso a la energía eléctrica, se han utilizado los datos de consumos anuales facturados por las prestadoras a nivel de Departamento, total de usuarios por Departamento y el cuadro tarifario vigente en el cuarto trimestre del año en curso. Esta información es comunicada anualmente por las empresas prestadoras del servicio a la Secretaría de Energía de la Nación, quien, a su vez, las utiliza para la elaboración de estadísticas y su publicación. Los costos y consumos relativos al acceso a gas han sido estimados a partir de la consulta a la base de datos del Ente Nacional Regulador del Gas (ENARGAS). Se utilizaron las tablas de gas residencial entregado, según mes del año, cantidad de usuarios y cuadro tarifario. Esta información sólo se encuentra disponible a nivel de Provincias. No obstante, considerando que las redes de gas cubren principalmente zonas urbanas, se ha empleado un valor único para los Aglomerados Urbanos de una misma provincia. Asimismo, algunos Aglomerados Urbanos no disponen de acceso al gas de red, para estos casos se estimó el costo mensual como el valor de una garrafa de $10 \mathrm{Kg}$ con subsidio, según el periodo considerado.

\section{Resultados}

La evolución de los valores del índice de pobreza energética muestra su descenso desde el año 2003 al 2015 (Grafico 1). Las variaciones anuales observadas corresponden con diferencias en la relación entre el costo de acceso a la energía y el ingreso total familiar, siendo mayor esta relación para 
el segundo y tercer trimestre. Este efecto puede estar asociado a una mayor demanda energética durante los meses fríos y la puesta en efecto de los nuevos cuadros tarifarios. Con respecto al intervalo analizado, la caída del índice varía de acuerdo al periodo considerado, desde 2003 a 2008 se observa un descenso pronunciado (de $40 \%$ a $10 \%$ en los meses más frios), desde 2008 a 2012 un descenso moderado (de $10 \%$ a 3 \%) y finalmente desde el año 2012 a 2015 una merma del 3\% al 0,8\% de hogares en situación de pobreza energética. Luego de establecer la quita a los subsidios para el consumo residencial de energía, el porcentaje aumenta a un $15,1 \%$ para los meses más fríos del año 2016, es decir, valores similares a los del año 2006.

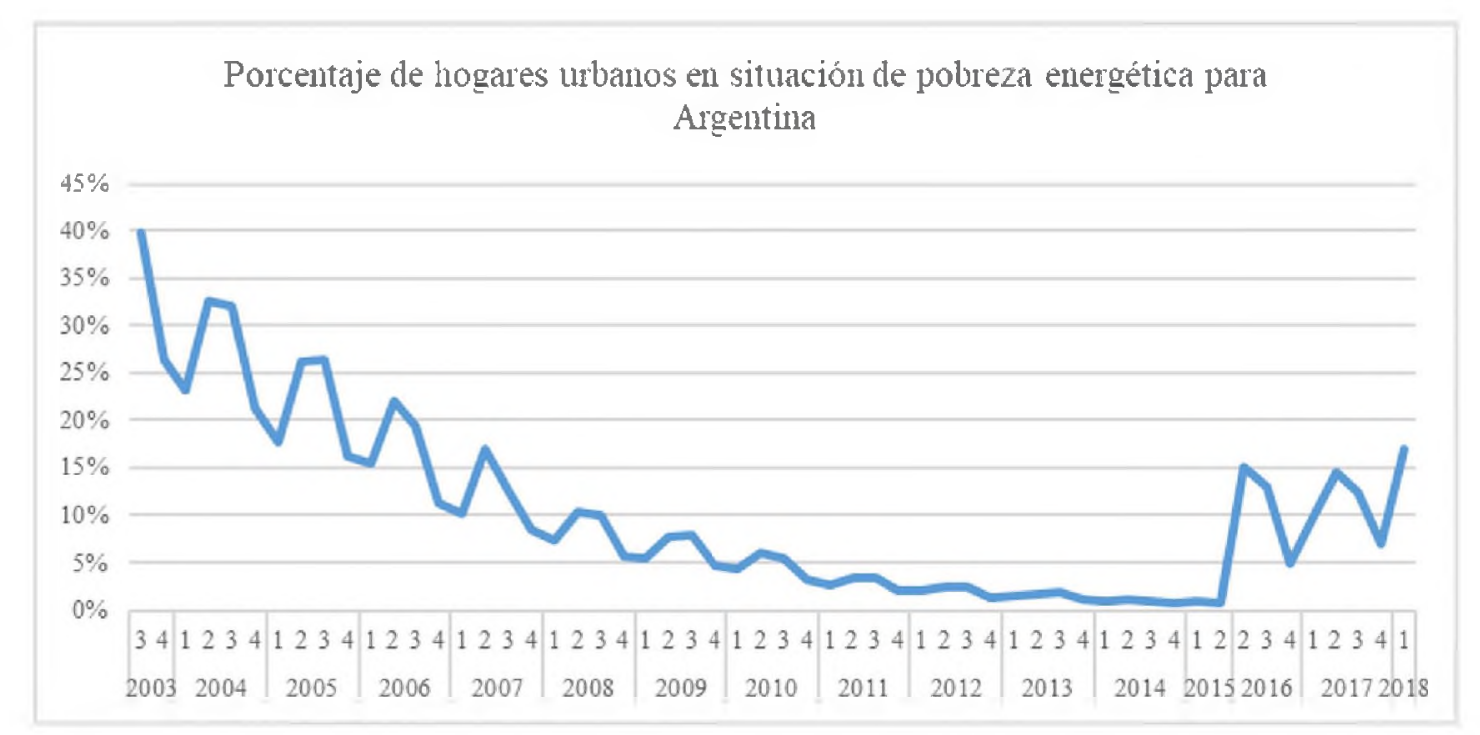

Gráfico 1 - Evolución del índice de pobreza energética para Argentina

El gráfico 2 indica la proporción de hogares pobres energéticos de acuerdo al decil de ingreso al que pertenecen. Desde la salida de la crisis argentina y hasta el año 2015, la pobreza energética pasó de ser un problema que representaba a la totalidad de los hogares situados en los primeros tres deciles de ingreso en el año 2003 (100\% para los dos primeros y 78\% del tercero), a estar localizada mayormente en el $8 \%$ de los hogares pertenecientes al primer decil en el año 2015. Así, para los años del periodo 2003 - 2005 el total de hogares pertenecientes al primer decil de ingreso y más del 80\%, correspondientes al segundo, están en situación de PE; en ese mismo periodo hay presencia de hogares PE hasta en el cuarto decil. En un segundo periodo de recuperación, comprendido desde el año 2006 al 2010 se reduce considerablemente la participación en deciles mayores al primero (pasando del 95\% y $59 \%$ de hogares pertenecientes al primer y segundo decil respectivamente, al $41.2 \mathrm{y}$ 
2\%). En un tercer periodo, desde el año 2012 al 2015, la totalidad de hogares en situación de pobreza energética corresponden solo al primer decil y son menores al $10 \%$ del total. Comparativamente, el cambio en la distribución de hogares en situación de PE entre el año 2015 a 2016 resulta cuando menos abrupto. Argentina pasó de tener solo un $6,5 \%$ de hogares en situación de PE, representada mayormente en el primer decil de ingreso, a un $78 \%$ y $23 \%$.

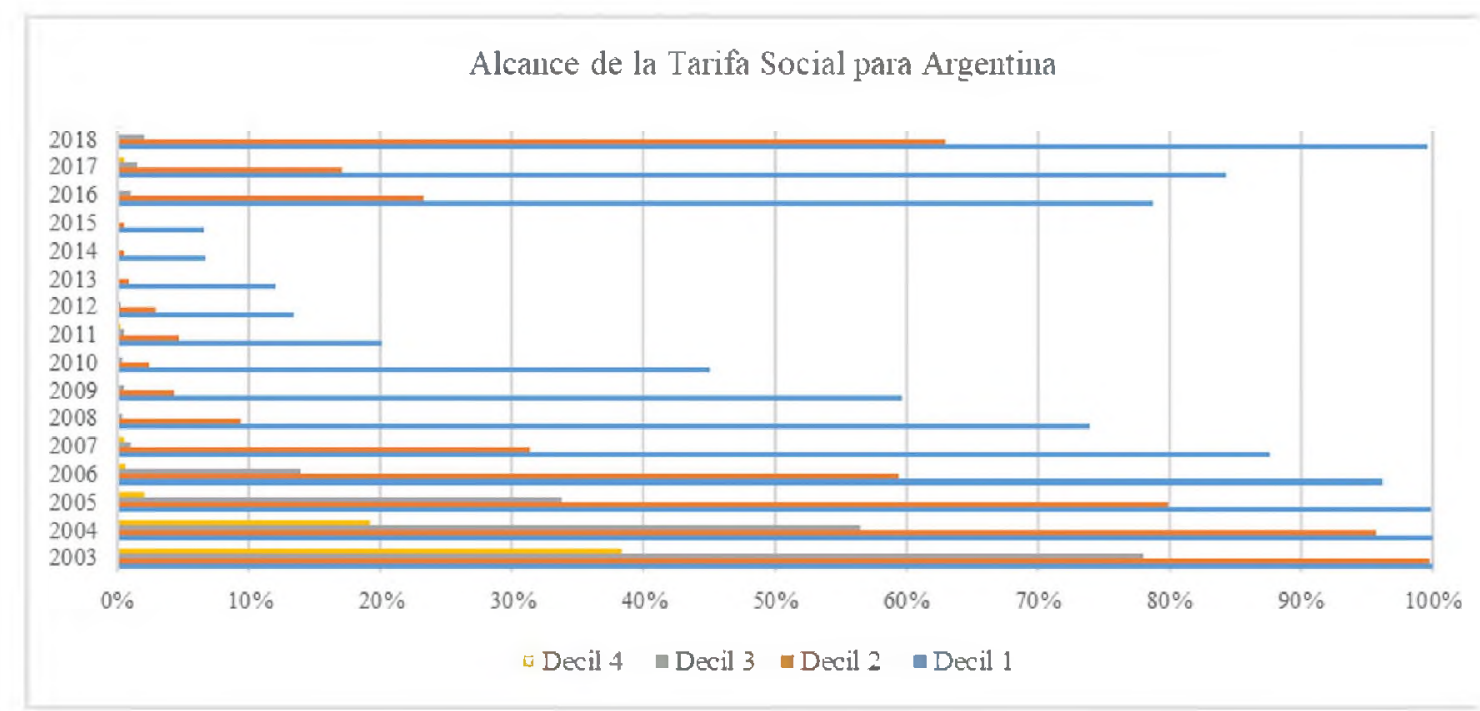

Gráfico 2 - Presencia de hogares PE según decil de ingreso

En junio del año 2016, el gobierno inicia la aplicación de una tarifa diferencial, definida para hogares en situación de vulnerabilidad económica, la cual recibe el nombre de "Tarifa Social". Las cargas tarifarias para esta nueva categoría se encuentran parcialmente subsidiadas; no obstante, si se comparan con los costos de la tarifa vigente hasta 2015, resultan más caras.

El no haber definido la política de subsidio tarifario a partir de un concepto como el de pobreza energética, implicó una importante limitación del alcance de la práctica. De hecho, puede observarse en el gráfico 4.12 que históricamente los requerimientos establecidos para el acceso a la tarifa social cubren a la menor parte de los hogares en situación de PE. Considerando el hipotético caso de que todos los jefes de hogar sean titulares del servicio (curva en naranja), solo entre los años 2006 y 2010 la aplicación alcanzaría a más del $40 \%$ de los jefes de hogar afectados por la PE. Si se tiene en cuenta que los jefes de hogar, que no son propietarios de la vivienda que ocupan, no tienen la titularidad del servicio, los valores caen un $10 \%$ en promedio para todo el periodo. Si bien el nuevo esquema tarifario fue impuesto recién a mediados de 2016, es evidente que, a partir del análisis de la evolución de 
los hogares en situación de pobreza energética durante los últimos años, los requerimientos nunca hubieran cubierto al grueso de la población más vulnerable.

Ahora, si se evalúa el alcance de la tarifa social solamente en el año en que fue impuesta, 2016, se observa que no alcanza al 50\% de los jefes de hogar en situación de $\mathrm{PE}$, aun suponiendo que el jefe de hogar en situación de PE sea titular del servicio. Alternativamente, si se considera que los jefes de hogar no tienen la titularidad, solamente un $41 \%$ son cubiertos por la tarifa social.

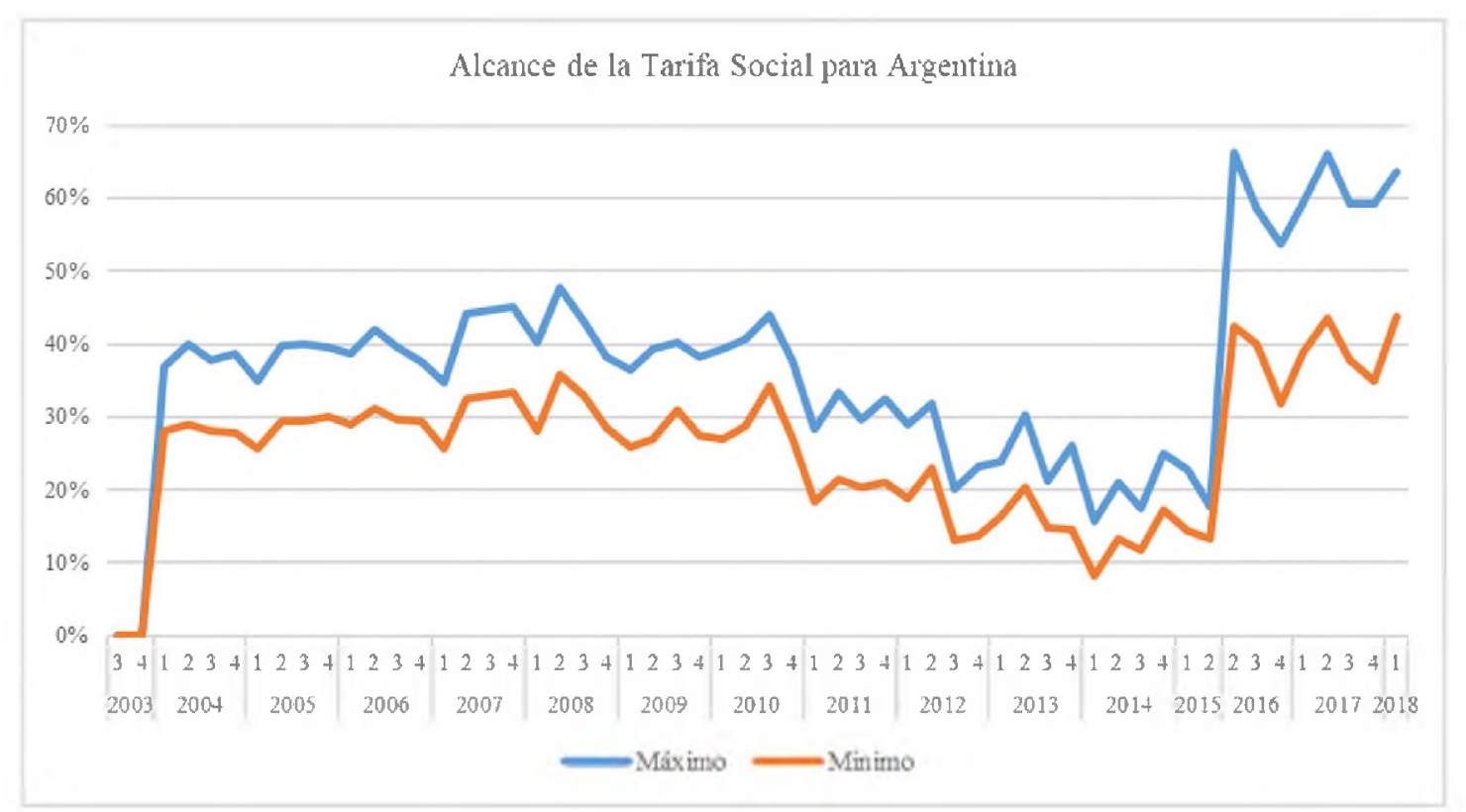

Gráfico 3 - Alcance de la tarifa social sobre hogares en situación de PE.

\section{Conclusiones}

Se ha estimado un indicador de Pobreza Energética para los hogares de los Aglomerados Urbanos de Argentina. Este indicador está basado en la definición de pobreza energética a partir de la comparación entre los ingresos y gastos totales que un hogar dispone para poder acceder a las fuentes de energía secundaria disponibles de manera residencial. Aquellos hogares que disponen de más de un $10 \%$ de sus ingresos totales son considerados en situación de Pobreza Energética.
Este trabajo da cuenta de que, aún al haber propuesto cambios tarifarios escalonados, la proporción de hogares en situación de PE se disparó entre 2015 y 2016. El cambio tarifario apunta a generar un cambio en el consumo de la energía; no obstante, por la conformación material de la vivienda y sus particularidades térmicas, el problema de consumo no puede ser definido como meramente cultural o fundamentado en la estructuración de un comportamiento de consumo 
No obstante, es provechoso observar la evolución del índice de Pobreza Energética desde uno de los periodos de crisis económica e institucional más graves sufridos en la historia argentina, como lo fue el iniciado en diciembre del año 2001. La comparación permite visibilizar una importante mejora de la situación, llegando a niveles inferiores al 3\% entre el año 2012 y 2015. Asimismo, el cambio entre el año 2015 y 2016 retrotrae los niveles de Pobreza Energética a los del año 2006 - 2007. Siendo, para el primer trimestre del 2018, de un 17\% para Argentina.

La conceptualización de la Tarifa Social, como política de desarrollo social, responde al esquema de las políticas de desarrollo humano de finales de los años 80's. Éste busca establecer poblaciones foco que serían afectadas directamente por la política, que se supone, está definida a su medida. Conceptualmente, la Tarifa Social ha sido definida por el gobierno como un "beneficio", una ayuda para aquellos jefes de hogar que, estando registrados en el sistema de ANSES y cumpliendo una serie de condiciones relativas a una situación de vulnerabilidad, es automáticamente otorgada. Los conceptos de "beneficio" y "ayuda" parten de una lógica asimétrica; el término economicista de "beneficio" implica la ganancia o el provecho que se obtiene a partir de una inversión y, en el contexto de la eco- nomía de capital, cobra forma en la lógica unidireccional materializada en relaciones desiguales. Por otro lado, el concepto de "ayuda" implica la dependencia de un hogar con respecto a las políticas dispuestas desde el Estado. Al ser parcial, el otorgamiento de un beneficio, como la Tarifa Social, es correctamente definido como una "ayuda", un descuento que brinda el Estado a un jefe de hogar para hacer más soportable su situación de carencia.

A pesar de haber sido definida de manera focal, en términos de su alcance, la aplicación de la Tarifa Social cubre a menos de la mitad de los hogares en situación de PE. La definición de las condiciones que han sido establecidas para el otorgamiento del beneficio no logra identificar el universo de hogares situados bajo la problemática, lo cual refiere o al desconocimiento de la situación de los hogares en situación de $\mathrm{PE}$ o a la negligencia en la acción. A1 tener como objetivo únicamente al jefe de hogar se ha evitado la contextualización de la situación de pobreza en términos del núcleo familiar, lo cual brindaría elementos para aumentar el alcance de la aplicación. Asimismo, se supone que el jefe de hogar es titular del servicio, lo cual no siempre es correcto, por lo que un número importante de hogares, que alquilan la vivienda o que la ocupan de manera prestada no siempre disponen de la titularidad del servicio. 


\section{Bibliografía}

Bazilian, M., Nakhooda, S., Van de Graaf, T., 2014. Energy governance and poverty. Energy Res. Soc. Sci. 1, 217-225.

Bouzarovski, S., Petrova, S., Sarlamanov, R., 2012. Energy poverty policies in the EU: A critical perspective. Energy Policy 49, 76-82.

González-Eguino, M., 2015. Energy poverty: An overview. Renew. Sustain. Energy Rev. 47, 377-385.
Nussbaumer, P., Bazilian, M., \& Modi, V. (2012). Measuring energy poverty: Focusing on what matters. Renewable and Sustainable Energy Reviews, 16(1), 231-243.

Okushima, S., 2016. Measuring energy poverty in Japan, 2004-2013. Energy Policy 98, 557-564. doi:10.1016/j.enpol.2016.09.005

Pachauri, S., \& Spreng, D. (2004). Energy use and energy access in relation to poverty. Economic and Political weekly, 271-278. 